\title{
The Magnetic Optical Rotation of Carbonyl Compounds
}

\author{
BY J. WINKLER \\ (Arbeitsgemeinschaft für Strukturchmie, Technische Hochschule, 8 München 2, Steinheilstrasse 1, Germany)
}

Recently McCaffery et al. ${ }^{1}$ examined the magnetic optical activity of several ketones and stated that the $n \rightarrow \pi^{*}$ transition of almost all the compounds studied showed no magnetic c.d. These results contrast with earlier measurements of magnetic o.r.d. by Shashoua ${ }^{2}$ and Briat, ${ }^{3}$ who in some of these compounds observed the presence of $A$ terms, which are larger by a factor of 100 than the

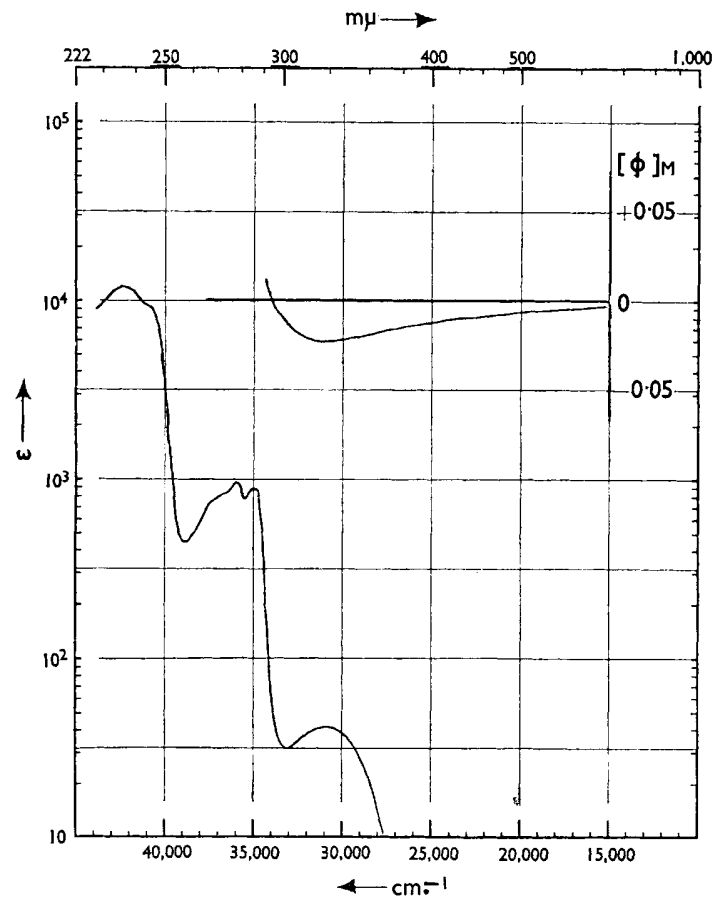

FIGURE 1. Acetophenone, ultraviolet spectrum and magnetic optical rotatory dispersion; $[\phi]_{\mathrm{M}}$ is the molar rotation per unit magnetic field.

limit of sensitivity of McCaffery's apparatus. McCaffery $e t$ al. have therefore expressed doubts concerning the accuracy of Shashoua and Briat's experimental findings.

We have observed that errors do appear in magnetic o.r.d. measurements if, due to reflections in the sample cell, part of the light passes through the cell several times. Contrary to natural optical activity, the rotation of the plane of polarisation will thus be increased. In the case of an absorbing sample, the reflected light is reduced and a deviation from the baseline (which had been registered with the transparent solvent) results. It can be shown that this deviation is proportional to $\alpha_{0}\left(T^{2}-1\right), T$ being the transmission of the solution and $\alpha_{0}$ the magnetic o.r.d. of the sample cell filled with the solvent. The deviation has the opposite sign of $\alpha_{0}$. If one therefore follows the sign arrangement of Stephens



FIGURE 2. Benzophenone, ultraviolet spectrum and magnetic optical rotatory dispersion.

et al., ${ }^{4}$ the error will approximate to a positive $A$ term. The magnitude of the error depends on the geometrical-optical characteristics of the measuring instrument. For our apparatus ${ }^{5}$ it amounts to $c a \cdot 0 \cdot 02^{\circ}$ at a light frequency of 30,000 $\mathrm{cm} .^{-1}$, a magnetic field strength of $7.5 \mathrm{~kg}$, and sample cells of $0.25 \mathrm{~cm}$. path length. Therefore, the magnitude, sign, and shape of the curves measured by Shashoua and Briat for aliphatic ketones may be explained by this error, taking into account the fact that they used the opposite sign definition. 
We could however, confirm Shashoua's measurements of acetophenone (see Figure 1) which are in agreement with the findings of McCaffery et al. The $B$ term of the $n \rightarrow \pi^{*}$ transition cannot be determined owing to the low sensitivity of our instrument. The positive increase from 31,000 $\mathrm{cm} .^{-1}$ can be explained by the positive rotation of the $B$ term of the $\pi \rightarrow \pi^{*}$ transition and the negative rotation at lower frequencies by a strongly negative effect in the far u.v. region. Thus the minimum at $31,000 \mathrm{~cm} \cdot .^{-1}$ is not due to a Cotton effect and lies only accidentally near the point of the $n \rightarrow \pi^{*}$ transition. However, we could not detect the small effect at $27,000 \mathrm{~cm} .^{-1}$, which Shashoua attributes to the singlet $\rightarrow$ triplet transition of the acetophenone. Benzophenone (Figure 2) shows a similar curve to acetophenone, so that the interpretation of this spectrum is analogous.

We thank the Stiftung Volkswagenwerk for support.

(Received, April 8th, 1968; Com. 435.)

${ }^{1}$ A. J. McCaffery, G. N. Henning, P. N. Schatz, A. B. Ritchie, H. P. Perzanowski, O. R. Rodig, A. W. Norvelle, jun., and P. J. Stephens, Chem. Comm., 1966, 520.

2 V. E. Shashoua, J. Amer. Chem. Soc., 1964, 86, 2109.

${ }^{3}$ B. Briat, Compt. rend., 1964, 258, 2788.

4 P. J. Stephens, W. Suetaka, and P. N. Schatz, J. Chem. Phys., 1966, 44, 4592.

${ }^{5} \mathrm{~J}$. Winkler, $Z$. analyt. Chem., in the press. 